\title{
Phân tích các yếu tố ảnh hưởng tới việc hoàn thành dự án đầu tư theo hình thức đối tác công tư (PPP): Nghiên cứu trường họ̣p của Thành phố Hồ Chí Minh \\ Factors affecting the completion of public-private partnership (PPP) projects: Case study of Ho Chi Minh City
}

\begin{tabular}{|c|c|}
\hline \multicolumn{2}{|c|}{$\begin{array}{l}{ }^{1} \text { Đại học Tomas Bata in Zlín, Cộng hòa Séc } \\
\text { *Tác giả liên hệ, Email: trung.nt@ou.edu.vn }\end{array}$} \\
\hline THÔNG TIN & TÓM TẮT \\
\hline $\begin{array}{l}\text { DOI: } 10.46223 / \mathrm{HCMCOUJS} . \\
\text { econ.vi.15.1.249.2020 }\end{array}$ & $\begin{array}{l}\text { Hợp tác công tư được xem là một trong những giải pháp } \\
\text { tối ưu để giải quyêt các vần đề về ngân sách nhà nước, phát triển } \\
\text { cơ sở hạ tầng và nâng cao chất lượng dịch vụ công. Hình thức } \\
\text { này được chú trọng trong cả nghiên cứu và thực tiến tại các nước } \\
\text { phát triển, tuy nhiên vấn còn hạn chế tại các quốc gia đang phát } \\
\text { triển như Việt Nam. Nghiên cứu này nhằm tìm hiểu các yếu tố } \\
\text { ảnh hưởng tới việc hoàn thành dự án đầu tư theo hình thức đối }\end{array}$ \\
\hline Ngày nhận: 20/11/2019 & \\
\hline Ngày nhận lại: 17/12/2019 & được sử dụng để phân tích số liệu, bao gồm: Thống kê mô tả, \\
\hline Duyệt đăng: 21/01/2020 & $\begin{array}{l}\text { Kiểm định Cronbach's Alpha, Phân tích nhân tố khám phá EFA, } \\
\text { Chỉ số quan trọng tương đối (Relative Importance Index - RII). } \\
\text { Kết quả nghiên cứu cho thấy: Các nhóm yếu tố Quản lý rủi ro, } \\
\text { Hiệu quả trong quá trình thực hiện dự án, Tài chính dự án, và } \\
\text { Chính trị và môi trường kinh doanh đều tác động tới việc hoàn } \\
\text { thành dự án PPP tại TP.HCM. Nghiên cứu cũng chỉ ra và xếp }\end{array}$ \\
\hline $\begin{array}{l}\text { Tù khóa: } \\
\text { dự án PPP, hiệu quả dự án, } \\
\text { quản trị nhà nước }\end{array}$ & $\begin{array}{l}\text { hạng mức độ tác động của các yếu tố thuộc các nhóm yếu tố trên } \\
\text { trong phạm vi mâu nghiên cứu thu thập được. Từ kết quả trên, } \\
\text { tác giả đã đưa ra một số khuyến nghị chính sách cho các dự án } \\
\text { PPP đang thực hiện tại TP.HCM. }\end{array}$ \\
\hline
\end{tabular}

\section{ABSTRACT}

Public-private partnership is one of the best solutions to help save the government budget, develop the infrastructure and improve the quality of public services and has become a popular research topic in developed countries. However, there are few studies on this topic in developing countries like Vietnam. This study aims to understand the factors affecting the completion of public-private partnership projects (PPP projects) in Ho Chi Minh City (HCMC). Quantitative research methods are used for data analysis including Descriptive Statistics, Cronbach's Alpha 
Testing, Exploratory Factor Analysis (EFA) and Relative Importance Index (RII). The research results show that factors of Risk Management, Effectiveness in Project Implementation, Project Finance, and Politics and Business Environment all affect the completion of PPP projects in HCMC. The study also

Keywords:

PPP project, project performance, governance indicates and ranks the impact of factors in the above factor groups within the sample collected. The author accordingly made some policy recommendations for PPP projects being implemented in HCMC.

\section{Mở đầu}

Trong những năm gần đây, nền kinh tế Việt Nam luôn tăng trưởng ở tốc độ cao. Song song với mức tăng trưởng đó là những áp lực về tài chính lên Chính phủ về nguồn lực để duy trì đà tăng trưởng và xây dựng đất nước. Trong bối cảnh này, ngoài việc hoàn thiện những cơ chế tài chính cũng như điều tiết nguồn thu ngân sách hiệu quả hơn để khơi thông các nguồn lực, thì huy động nguồn vốn từ khu vực tư nhân dưới hình thức đầu tư theo mô hình hợp tác công tư (dự án PPP) cũng sẽ được xem là một trong những ưu tiên hàng đầu. Đối với Thành phố Hồ Chí Minh (TP.HCM), với nhu cầu đầu tư lớn và tiềm lực xã hội hóa đứng đầu cả nước, Chính quyền thành phố xác định PPP là một trong những giải pháp tối ưu để giải quyết các vấn đề về tiết kiệm ngân sách, phát triển đồng bộ kết nối hạ tầng và tạo sự cạnh tranh mạnh mẽ giữa các thành phần kinh tế. Chính bởi vậy, việc phân tích các dự án PPP là một vấn đề cấp thiết nhằm tìm ra được mô hình hợp tác dài hạn, chia sẻ một cách hợp lý không chỉ là lợi ích mà cả rủi ro giữa các bên tham gia. Từ đó, có thể đưa ra những khuyến nghị về chính sách để hoàn thiện quá trình hợp tác PPP, giúp thu hút sự tham gia của khối tư nhân trong các lĩnh vực phát triển của quốc gia.

\section{Cơ sở lý thuyết và giả thuyết nghiên cứu}

\subsection{Cơ sở lý thuyết về đầu tư PPP}

PPP (Public - Private Partnership) được định nghĩa là một giao kết bằng hợp đồng giữa Nhà nước và tư nhân, theo đó hai bên chia sẻ các kỹ năng và tài sản của mỗi bên trong việc phân phối dịch vụ cho xã hội (European Commission, 2003). Cụ thể hơn, các quan hệ đối tác công tư được định nghĩa là các thỏa thuận mà theo đó Nhà nước ký hợp đồng dài hạn với khu vực tư nhân cho việc xây dựng, vận hành, quản lý các cơ sở hạ tầng, hoặc cung cấp dịch vụ công phục vụ cho mục đích cộng đồng (Grimsey \& Lewis, 2002). Theo Vassallo Magro và Bartolome (2010), mô hình hợp tác công tư PPP dựa trên bốn nguyên tắc cơ bản sau: (1) Là các hàng hóa, hoặc dịch vụ có lợi ích chung, có sự thất bại của thị trường và phải được giám sát bởi khu vực công; (2) Khu vực tư nhân có thể nâng cao hiệu quả và chất lượng trong việc phân bổ ngân sách công; (3) Có thể phân bổ rủi ro một cách hợp lý giữa khu vực công và khu vực tư nhân; (4) Sự tham gia của khu vực tư nhân trong suốt dự án có thể giúp nâng cao khả năng cung cấp hàng hóa và các dịch vụ công.

Hiện nay, PPP đang là một trong những hình thức đầu tư phát triển cơ sở hạ tầng được nhiều quốc gia sử dụng, đặc biệt là các quốc gia đang phát triển do chính phủ các quốc gia cần tiếp cận nguồn vốn và công nghệ từ phía khu vực tư nhân cho nhu cầu xây dựng đất nước. Walker 
và Smith (1995) đã đưa ra 3 lý do chính để sử dụng PPP, cụ thể: (1) Khu vực tư nhân có tính linh hoạt hơn khu vực công. Ví dụ như khu vực tư nhân không chỉ tiết kiệm được chi phí dự án trong quy hoạch, thiết kế, xây dựng và vận hành; mà còn tránh được nạn quan liêu và giảm bớt gánh nặng hành chính; (2) Khu vực tư nhân có thể cung cấp dịch vụ tốt hơn cho khu vực công, và thiết lập các mối quan hệ công - tư một cách cân bằng về cả vốn và rủi ro; (3) Khu vực công đôi lúc không có khả năng huy động vốn lớn cho các dự án cơ sở hạ tầng quy mô lớn, và sự tham gia của tư nhân có thể làm giảm gánh nặng tài chính của Chính phủ. Ghobadian, O'Regan, Gallear, và Viney (2004) còn bổ sung thêm hai lý do nên sử dụng rộng rãi hơn các dự án PPP. Thứ nhất, khu vực tư nhân có thể tìm hiểu rõ hơn nhu cầu của khách hàng trong mọi thời điểm; và thứ hai, khu vực tư nhân có khả năng cung cấp các kỹ năng về công nghệ, kiến thức, từ đó có thể dẫn đến chất lượng dịch vụ tốt hơn.

Đối với Việt Nam, một nghiên cứu của Huynh (2012) về hình thức hợp tác công tư để phát triển cơ sở hạ tầng giao thông đường bộ chỉ ra rằng đối với sự thành công của $\mathrm{PPP}$, quan trọng nhất là cả hai khu vực cần điều chỉnh sự khác biệt về mục tiêu đầu tư trên cơ sở đảm bảo kết quả hợp tác là hợp lý cho cả hai phía, bình đẳng giữa các bên.

\subsection{Lập luận giả thuyết}

\subsubsection{Các yếu tố pháp lý và việc hoàn thành dụ án PPP}

Các yếu tố pháp lý được đánh giá là ảnh hưởng tới việc hoàn thành dự án PPP trên thế giới (Hardcastle, Edwards, Akintoye, \& Li, 2005; Ismail \& Ajija, 2012; Yescombe, 2007; Yitmen, Akiner, \& Tekce, 2012). Trong đó, Hardcastle và cộng sự (2005) cho rằng khả năng thực hiện dự án PPP phụ thuộc chủ yếu vào khung pháp lý thuận lợi, và khung pháp lý cho phép dự án được phát triển mà không có quá nhiều giới hạn về mặt luật pháp không cần thiết về sự tham gia của khu vực tư nhân. Các dự án PPP thực hiện trong môi trường có khung pháp lý phù hợp cũng thường thu hút nguồn tài chính cho dự án tốt hơn (Yescombe, 2007). Ngoài ra, Yitmen và cộng sự (2012) cũng thực hiện nghiên cứu để phát triển một khung phân tích khả năng áp dụng các cơ chế PPP ở Thổ Nhĩ Kỳ và kết luận rằng khuôn khổ chính trị và pháp lý thuận lợi cũng là một trong những yếu tố ảnh hưởng tới việc hoàn thành dự án.

Sự hỗ trợ ở khía cạnh pháp lý và xây dựng được các quy định về pháp luật giúp các dự án PPP có thể được đảm bảo đầu tư dài hạn, giảm chi phí giao dịch, tránh các vấn đề tranh chấp trong thời điểm thực hiện dự án, đồng thời cũng thu hút các nhà đầu tư tư nhân hơn. Theo Yitmen và cộng sự (2012), khung pháp lý về dự án PPP cần bao gồm: (1) Khung pháp lý thuận lợi, cụ thể là có các luật hoặc điều khoản có thể giúp áp dụng khi đầu tư PPP và thực hiện các chức năng của nó, ví dụ như: Quyền hợp pháp thành lập doanh nghiệp dự án, trách nhiệm của doanh nghiệp dự án khi ký kết với các nhà thầu phụ,... và (2) Khung thể chế mạnh mẽ, cụ thể là các điều khoản đảm bảo về mặt tài chính và vận hành lâu dài cho dự án, ví dụ như cam kết về việc đảm bảo hợp đồng dài hạn, cam kết giải ngân vốn đối ứng từ phía Nhà nước,....

Như vậy, giả thuyết được đặt ra như sau:

H1: Các yếu tố pháp lý tác động duong đến Việc hoàn thành dự án PPP

\subsubsection{Quản lý rủi ro và Việc hoàn thành dụ án PPP}

Quản lý rủi ro được nhận định là một trong những yếu tố ảnh hưởng tới việc hoàn thành dự án PPP (Akintoye, Craig, \& Eamon, 1998; B. Li, Akintoye, Edwards, \& Hardcastle, 2005; Li \& Zou, 2012). Những nghiên cứu nền tảng về quản lý rủi ro trong dự án PPP xuất phát từ 
Akintoye và cộng sự (1998) đối với lĩnh vực cơ sở hạ tầng, trong đó các rủi ro liên quan đến thiết kế, chi phí xây dựng, hiệu quả hoạt động, hợp đồng, chậm trễ tiến độ, chi phí hoạt động, tín dụng, những thay đổi của chính phủ và các rủi ro liên quan đến đất đai là những yếu tố ảnh hưởng tới việc hoàn thành dự án. Nghiên cứu của $\mathrm{B}$. Li và cộng sự (2005) về các dự án PPP tại Anh chỉ ra các rủi ro bao gồm: rủi ro do thay đổi chính sách, rủi ro kinh tế vĩ mô, rủi ro về pháp luật, rủi ro về xã hội, các rủi ro tự nhiên, các rủi ro tài chính, các rủi ro về vận hành, các rủi ro về mối quan hệ giữa hai bên và rủi ro do bên thứ ba gây ra. Các nghiên cứu sau dựa trên nghiên cứu của $B$. Li và cộng sự (2005) thường tập trung vào việc phân bổ rủi ro trong dự án $P P P$, theo đó Mouraviev (2012) khẳng định rằng rủi ro trong các dự án PPP phải được phân bổ cho cả khu vực nhà nước và khu vực tư nhân và duy trì được việc phân bổ này xuyên suốt thời gian từ lúc ký kết hợp đồng cho đến thời gian kết thúc.

Nhằm mục đích phân loại các rủi ro tồn tại trong dự án PPP, J. Li và Zou (2012) trong nghiên cứu về các dự án PPP tại Trung Quốc chia các rủi ro có thể xảy ra thành 6 nhóm, bao gồm: (a) Nhóm rủi ro liên quan đến giai đoạn nghiên cứu khả thi: rủi ro ô nhiễm môi trường, rủi ro thu hồi đất và bồi thường, rủi ro xảy ra các tranh chấp, rủi ro do thay đổi nhu cầu, rủi ro khi không có sự đồng thuận chính trị; (b) Nhóm rủi ro liên quan đến tài chính: rủi ro về thay đổi lãi suất, rủi ro lạm phát, rủi ro thay đổi pháp luật, rủi ro về sự ổn định của thị trường tài chính; (c) Nhóm rủi ro liên quan đến thiết kế: rủi ro lỗi thiết kế, nguy cơ điều chỉnh thiết kế quá nhiều; (d) Nhóm rủi ro liên quan đến xây dựng: có các phát sinh vượt quá ngân sách; chậm trễ về thời gian thi công, rủi ro tác động đến môi trường, rủi ro về chất lượng công trình, rủi ro về thời tiết và các điều kiện bất khả kháng, rủi ro về khả năng vỡ nợ của nhà thầu; (e) Nhóm rủi ro liên quan đến vận hành: rủi ro khi doanh thu không đảm bảo, rủi ro về chi phí hoạt động kinh doanh, rủi ro về thay đổi pháp luật, rủi ro về năng suất thấp và các rủi ro vỡ nợ; (f) Nhóm rủi ro liên quan đến việc chuyển giao: rủi ro giá trị còn lại thấp, rủi ro khi chuyển giao thất bại.

Như vậy, giả thuyết được đặt ra như sau:

\section{H2: Các yếu tố Quản lý rủi ro tác động dương đến Việc hoàn thành dụ án PPP}

\subsubsection{Hiệu quả trong quá trình chuẩn bị dụ án và Việc hoàn thành dụ án PPP}

Nhằm đo lường việc hoàn thành dự án PPP, Yuan, Wang, Skibniewski và Li (2011) nghiên cứu 48 yếu tố và đã chỉ ra rằng cự yếu tố bao gồm: Đấu thầu với giá cả phải chăng; Thiết kế, lập kế hoạch và giai đoạn lập kế hoạch bởi khu vực công; Hiệu quả và kiểm soát quá trình trong khu vực tư nhân; và Mức độ hài lòng cho các bên tham gia dự án là các yếu tố ảnh hưởng mạnh mẽ đến hiệu quả trong quá trình thực hiện dự án. Nghiên cứu của Tang, Shen, Skitmore, và Cheng (2013) cũng xác định các yếu tố hiệu quả trong quá trình thực hiện dự án cũng có ảnh hưởng mang tính quyết định xem dự án PPP có thể hoàn thành hay không, kết hợp với kết quả nghiên cứu của Yitmen và cộng sự (2012) đối với các dự án PPP tại Thổ Nhĩ Kỳ, có thể đánh giá rằng ở góc nhìn của Chính phủ, các dự án PPP đạt hiệu quả phải đáp ứng được: (a) Thiết kế xây dựng phải dễ hiểu, rõ ràng và đầy đủ thông tin; (b) Ứng dụng công nghệ mới và các sáng tạo trong xây dựng; (c) Cân bằng được các yêu cầu về thiết kế với ngân sách sẵn có; (d) Có sự nâng cao trong các yêu cầu kỹ thuật, và yêu cầu để lựa chọn tư vấn, lựa chọn chuyên gia cũng như nhà thầu. Ở góc nhìn của tư nhân, dự án PPP đạt hiệu quả trong quá trình thực hiện phải: (a) Biết cách phân phối hoặc thuê ngoài các khối lượng công việc cho các nhà thầu phụ để có thể chuyên biệt hơn; (b) Thiết lập được mạng lưới/mối quan hệ mạnh dưới sự điều phối của nhà thầu chính; (c) Có động lực thực hiện các dự án; (d) Lập kế hoạch hợp lý, có sự ủy quyền nhiều hơn cho người đại diện trực tiếp điều hành trong dự án. 
Như vậy, giả thuyết được đặt ra như sau:

H3: Các yếu tố Hiệu quả trong quá trình chuẩn bị dụ án tác động duơng đến Việc hoàn thành dụ án PPP

\subsubsection{Tài chính dụ án và Việc hoàn thành dụ án PPP}

Nghiên cứu của Zhang (2005) và $\mathrm{B}$. Li và cộng sự (2005) được xem như là nền tảng đối với nhóm yếu tố tài chính trong dự án $\mathrm{PPP}$, trong đó chú trọng vào tài chính và doanh thu dự kiến của dự án. $\mathrm{B}$. Li và cộng sự (2005) chỉ ra rằng: có sự tham gia của các tập đoàn tư nhân với tiềm lực tài chính mạnh và tốt; phía khu vực công và khu vực tư có sự phân bổ rủi ro thích hợp và thị trường tài chính sẵn có là những yếu tố quan trọng nhất ảnh hưởng đến các dự án PPP thành công ở Anh.

Cũng sử dụng khung phân tích của Li và cộng sự, các nghiên cứu của Cheung, Chan, Kajewski (2009) và Ismail (2013) về tác động của yếu tố tài chính đối với việc hoàn thành dự án PPP tại Trung Quốc và Malaysia. Theo đó, các yếu tố liên quan tới tài chính dự án được xác định là ảnh hưởng tới việc hoàn thành dự án PPP bao gồm: (a) Mở ra cơ hội kinh doanh mới cho khu vực tư nhân; (b) Khả năng bảo trì, vận hành dự án; (c) Khả năng tiết kiệm trong tổng chi phí dự án; (d) Giải quyết các vấn đề hạn chế về ngân sách nhà nước; (e) Khả năng chuyển giao công nghệ cho các công ty trong nước.

Như vậy, giả thuyết được đặt ra như sau:

H4: Các yếu tố Tài chính dự án tác động duơng đến Việc hoàn thành dự án PPP

\subsubsection{Chính trị và môi truò̀ng kinh doanh và Việc hoàn thành dụ án PPP}

Chan, Lam, Chan, Cheung, và Ke (2010) nghiên cứu về mối quan hệ giữa chính trị và môi trường kinh doanh với các dự án PPP tại Trung Quốc và chỉ ra rằng (a) Môi trường kinh tế vĩ mô ổn định; (b) Chia sẻ trách nhiệm giữa khu vực nhà nước và khu vực tư nhân; (c) Quá trình đấu thầu minh bạch và hiệu quả; (d) Môi trường chính trị và xã hội ổn định, và (e) Sự kiểm soát khôn khéo của chính phủ là những yếu tố ảnh hưởng tới việc hoàn thành dự án PPP. Cũng sử dụng mô hình phân tích này, Ismail và Ajija (2012) phát hiện ra rằng: Quản trị tốt; Các cam kết của Nhà nước và tư nhân; Khung pháp lý thuận lợi; Chính sách kinh tế tốt là quan trọng nhất trong việc thực hiện các dự án PPP ở Malaysia và cho kết quả tương đối giống nhau khi nghiên cứu với mẫu nghiên cứu ở các nước trên thế giới.

Như vậy, giả thuyết được đặt ra như sau: dụ án PPP

H5: Các yếu tố chính trị và môi trương kinh doanh tác động duơng đến Việc hoàn thành 


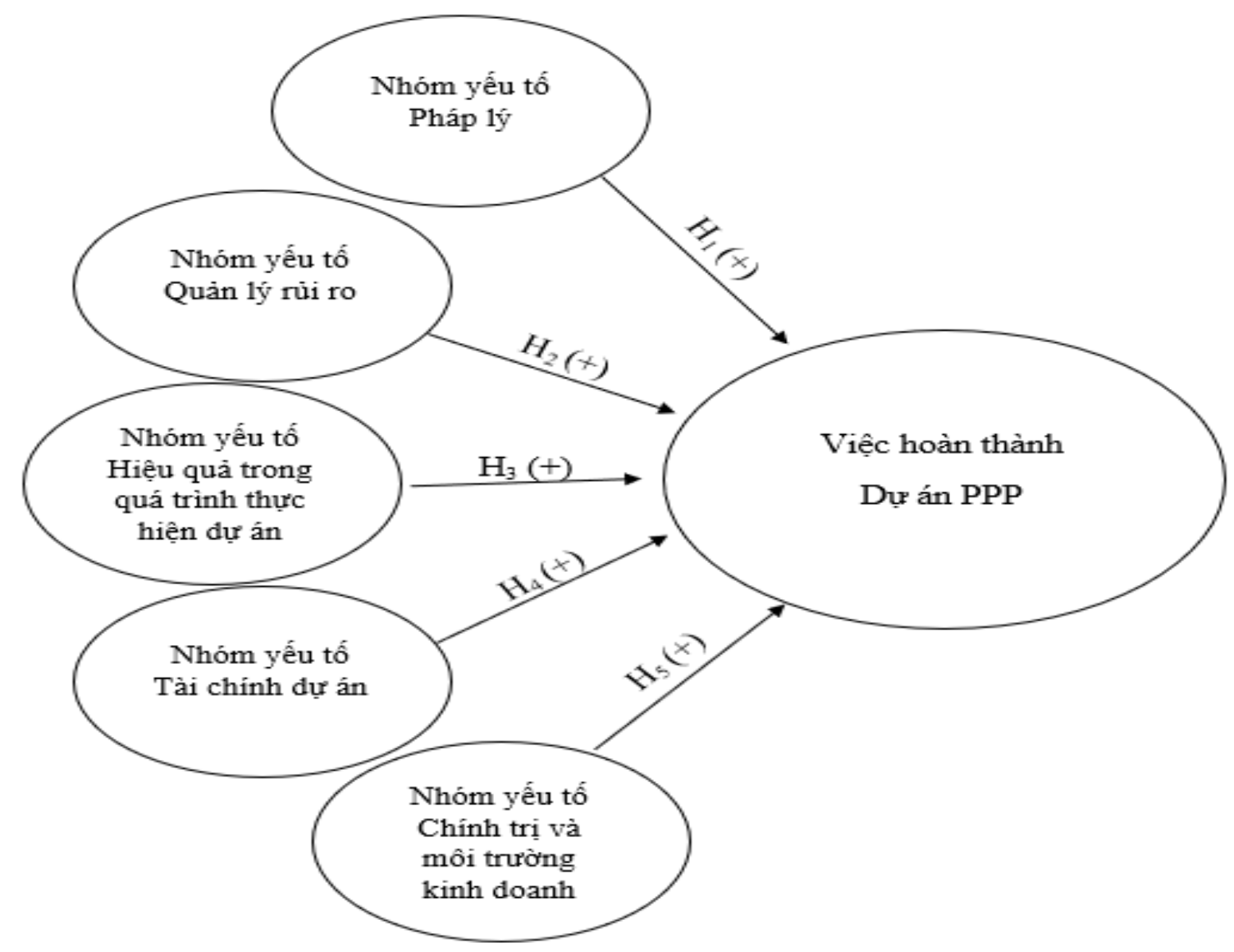

Hình 1. Mô hình nghiên cứu đề xuất

\section{Thiết kế nghiên cứu}

\subsection{Thang đo và mã hóa}

\section{Các yếu tố Pháp lý}

Nhóm yếu tố pháp lý sử dụng thang đo của Yitmen và cộng sự (2012). Thang đo gồm 2 biến quan sát: Khung pháp lý thuận lợi (PL1); và Khung thể chế mạnh mẽ (PL2).

\section{Các yếu tố Quản lý rủi ro}

Nhóm yếu tố Quản lý rủi ro sử dụng thang đo của $B$. Li và cộng sự (2005). Thang đo gồm 3 biến quan sát: Các rủi ro trong quá trình xây dưng dụ án, ví dụ nhu thiết kế không phù hợ, trê̂ tiến độ, thay đổi thiết kế, thay đổi nhà thầu (RR1); Các rủi ro về tài chính, bao gồm lãi suất, thay đổi tỷ giá, lạm phát,...(RR2); Các rủi ro giảm lọi nhuận đối với khu vục tư nhân (RR3).

\section{Hiệu quả trong quá trình chuẩn bị dụ án}

Nhóm yếu tố Hiệu quả trong quá trình thực hiện dự án sử dụng thang đo của Yitmen và cộng sự (2012). Thang đo gồm 5 biến quan sát: Các thiết kế rõ ràng, dêe hiểu và đủ thông tin cho các bên thực hiện (HQ1); Áp dụng các công nghệ xây dựng mới (HQ2); Chất lượng của các nhà tu vấn và nhà thầu thực hiện dụ án (HQ3); Mức độ chi tiết của việc lập kế hoạch (HQ4); Có sự hỗ trợ của các bên tham gia trong quá trình phát triển dụ án (HQ5). 


\section{Tài chính dư án}

Nhóm yếu tố Tài chính dự án sử dụng thang đo của Zhang (2005). Thang đo gồm 5 biến quan sát: Mở ra co hội kinh doanh mới cho khu vực tư nhân (TC1); Khả năng bảo trì, vận hành dụ án (TC2); Khả năng tiết kiệm trong tổng chi phi dụ án (TC3); Giải quyết các vấn đề hạn chế về ngân sách nhà nước (TC4); Khả năng chuyển giao công nghệ cho các công ty trong nước (TC5).

\section{Chính trị và môi trưòng kinh doanh}

Nhóm yếu tố Chính trị và môi trường kinh doanh sử dụng thang đo của Zhang (2005). Thang đo gồm 5 biến quan sát: Khả năng quản trị công của Chính phủ (CTMT1); Động lực và sụ hồ trọ của Chính phủ (CTMT2); Sụ trung thục trong thục hiện dụ án (TC3); Áp lục về mặt chính trị của các bên liên quan (CTMT4); Các vấn đề xã hội và sự ủng hộ của công đồng (CTMT5).

\subsection{Phương pháp nghiên cúu}

\subsubsection{Nghiên cúu định tính}

Nghiên cứu định tính được tiến hành nhằm hiệu chỉnh các biến quan sát không phù hợp với địa điểm nghiên cứu cũng như hiệu chỉnh từ ngữ trong bảng hỏi thông qua việc tham khảo ý kiến của những người tham gia vào sự hoàn thành của dự án PPP tại TP.HCM. Ngoài ra, bảng hỏi còn để hai câu hỏi mở nhằm thu thập thêm ý kiến của đối tượng được khảo sát.

\subsubsection{Nghiên cúu định lượng}

Các phương pháp phân tích định lượng được sử dụng bao gồm phương pháp thống kê mô tả, kiểm định Cronbach's Alpha, phân tích nhân tố khám phá EFA, phân tích chỉ số quan trọng tương đối (Relative Importance Index - RII). Dư liệu được xử lý bằng phần mềm Microsoft Excel 2016 và IBM SPSS 23.

\section{Kết quả nghiên cứu và bình luận}

\subsection{Mô tả mẫu nghiên cứu}

Nghiên cứu thực hiện khảo sát những người đang làm việc tại các tổ chức công và tổ chức tư liên quan tới dự án PPP và đã tham gia vào sự hoàn thành của dự án PPP tại TP.HCM, thời gian khảo sát từ tháng 12/2017 đến tháng $05 / 2018$. Khảo sát bao gồm việc khảo sát trực tiếp các đối tượng trực tiếp và gửi email khảo sát thông qua công cụ Google Form đối với các đối tượng không thể tiếp xúc trực tiếp. Tổng số phiếu được phát ra là 140 phiếu, tỷ lệ hồi đáp đạt $87,14 \%$ (122 phiếu). Sau khi tiến hành chọn lọc những phiếu đạt yêu cầu về hình thức (đủ số lượng câu trả lời, ...) số lượng phiếu đưa vào phân tích là 108 phiếu.

Theo đó, đối tượng khảo sát từ Khu vực công chiếm 54,63\%, đối tượng khảo sát từ Khu vực tư chiếm 45,37\%. Về vai trò của đối tượng khảo sát trong dự án, các đối tượng Quản lý nhà nước chiếm $50,93 \%$, Nhà đầu tư chiếm $38,89 \%$, Nhà tư vấn độc lập chiếm 4,63\%, Nhà nghiên cứu, chuyên gia chiếm $3,7 \%$ và Vai trò khác chiếm $1,85 \%$.

Các lĩnh vực PPP mà đối tượng khảo sát quan tâm tập trung chính vào các dự án Cơ sở hạ tầng, giao thông (cầu, đường, hầm...) với 75\%, dự án Tài nguyên môi trường (xử lý rác, nước sạch,...) chiếm 12,04\%, dự án Y tế, giáo dục chiếm 8,33\%, dự án Văn hóa, thể thao, du lịch chiếm 3,7\% và Lĩnh vực khác chiếm $0,93 \%$. 


\subsection{Kiểm định độ tin cậy thang đo}

Từ các kết quả thu thập được, nghiên cứu tính hệ số Cronbach's Alpha để loại các biến quan sát không đạt yêu cầu, hay các thang đo chưa đạt yêu cầu trong phân tích ban đầu. Theo Hair, Black, Babin, Anderson, và Tatham (2006), các biến quan sát có hệ số tương quan biến tổng bé hơn 0,3 sẽ bị loại bỏ và chỉ lựa chọn các biến quan sát có độ tin cậy Alpha từ 0,6 trở lên.

Kết quả kiểm định Cronbach's Alpha của thang đo Nhóm yếu tố pháp lý cho kết quả hệ số tương quan biến - tổng là 0,301 lớn hơn 0,3 , tuy nhiên hệ số Cronbach's Alpha là $0,462<$ 0,6 ; Như vậy kết luận thang đo Nhóm yếu tố pháp lý là không phù hợp, và không sử dụng cả 2 biến quan sát cho phân tích nhân tố khám phá EFA. Đối với thang đo này, số lượng câu hỏi chỉ là 2 câu hỏi và như vậy có thể nhận định rằng thang đo này không phù hợp để sử dụng khi nghiên cứu đối với trường hợp các dự án PPP tại TP.HCM.

Kết quả kiểm định Cronbach's Alpha của thang đo Nhóm yếu tố Quản lý rủi ro cho kết quả Hệ số Cronbach's Alpha là $0,824>0,6$; hệ số tương quan biến - tổng lớn hơn 0,3 . Như vậy kết luận thang đo Nhóm yếu tố Quản lý rủi ro là phù hợp, và có thể sử dụng cả 3 biến quan sát cho phân tích nhân tố khám phá EFA.

Kết quả kiểm định Cronbach's Alpha của thang đo Nhóm yếu tố Hiệu quả trong quá trình thực hiện dự án cho kết quả Hệ số Cronbach's Alpha là $0,788>0,6$; hệ số tương quan biến - tổng lớn hơn 0,3 ; Như vậy kết luận thang đo Nhóm yếu tố Hiệu quả trong quá trình thực hiện dự án là phù hợp, và có thể sử dụng cả 5 biến quan sát cho phân tích nhân tố khám phá EFA.

Kết quả kiểm định Cronbach's Alpha của thang đo Nhóm yếu tố Tài chính dự án cho kết quả Hệ số Cronbach's Alpha là $0,653>0,6$; hệ số tương quan biến - tổng lớn hơn 0,3 . Như vậy kết luận thang đo Nhóm yếu tố Tài chính dự án là phù hợp, và có thể sử dụng cả 5 biến quan sát cho phân tích nhân tố khám phá EFA.

Kết quả kiểm định Cronbach's Alpha của thang đo Nhóm yếu tố Chính trị và môi trường kinh doanh cho kết quả Hệ số Cronbach's Alpha là $0,854>0,6$; hệ số tương quan biến - tổng lớn hơn 0,3 . Như vậy kết luận thang đo Nhóm yếu tố Chính trị và môi trường kinh doanh là phù hợp, và có thể sử dụng cả 5 biến quan sát cho phân tích nhân tố khám phá EFA.

\section{Bảng 1}

Hệ số Cronbach’s Alpha của thang đo các nhóm yếu tố

\begin{tabular}{|c|c|c|c|c|}
\hline Biến quan sát & $\begin{array}{l}\text { Trung bình thang } \\
\text { đo nếu loại biến }\end{array}$ & $\begin{array}{l}\text { Phương sai thang } \\
\text { đo nếu loại biến }\end{array}$ & $\begin{array}{l}\text { Tương quan } \\
\text { Biến - Tổng }\end{array}$ & $\begin{array}{l}\text { Hệ số Cronbach's } \\
\text { Alpha nếu loại biến }\end{array}$ \\
\hline \multicolumn{5}{|c|}{ Nhóm yếu tố Pháp lý } \\
\hline PL1 & 4.1111 & .530 & .301 & · \\
\hline PL2 & 4.1759 & .520 & .301 & . \\
\hline \multicolumn{5}{|c|}{ Hệ số Cronbach's Alpha: 0,462 } \\
\hline \multicolumn{5}{|c|}{ Nhóm yếu tố Quản lý rủi ro } \\
\hline RR1 & 8.1852 & 1.648 & .612 & .826 \\
\hline RR2 & 8.2407 & 1.512 & .622 & .829 \\
\hline
\end{tabular}




\begin{tabular}{|c|c|c|c|c|}
\hline Biến quan sát & $\begin{array}{l}\text { Trung bình thang } \\
\text { do nếu loại biến }\end{array}$ & $\begin{array}{l}\text { Phương sai thang } \\
\text { đo nếu loại biến }\end{array}$ & $\begin{array}{l}\text { Tương quan } \\
\text { Biến - Tổng }\end{array}$ & $\begin{array}{l}\text { Hệ số Cronbach's } \\
\text { Alpha nếu loại biến }\end{array}$ \\
\hline RR3 & 7.7963 & 1.622 & .847 & .622 \\
\hline \multicolumn{5}{|c|}{ Hệ số Cronbach's Alpha: 0,824 } \\
\hline \multicolumn{5}{|c|}{ Nhóm yếu tố Hiệu quả trong quá trình thực hiện dự án } \\
\hline HQ1 & 15.3426 & 6.283 & .624 & .741 \\
\hline HQ2 & 15.6296 & 5.768 & .539 & .757 \\
\hline HQ3 & 15.4167 & 5.554 & .579 & .743 \\
\hline HQ4 & 15.5556 & 5.315 & .594 & .739 \\
\hline HQ5 & 15.5370 & 5.840 & .528 & .760 \\
\hline \multicolumn{5}{|c|}{ Hệ số Cronbach’s Alpha: 0,788 } \\
\hline \multicolumn{5}{|c|}{ Nhóm yếu tố Tài chính dự án } \\
\hline $\mathrm{TC} 1$ & 15.2593 & 5.054 & .432 & .600 \\
\hline $\mathrm{TC} 2$ & 15.3333 & 4.542 & .327 & .644 \\
\hline TC3 & 15.1667 & 4.907 & .350 & .625 \\
\hline TC4 & 15.2500 & 4.040 & .461 & .574 \\
\hline TC5 & 15.2870 & 4.319 & .501 & .555 \\
\hline \multicolumn{5}{|c|}{ Hệ số Cronbach's Alpha: 0,653 } \\
\hline \multicolumn{5}{|c|}{ Nhóm yếu tố Chính trị và môi trường kinh doanh } \\
\hline CTMT1 & 15.9722 & 6.906 & .725 & .811 \\
\hline CTMT2 & 16.0370 & 6.186 & .662 & .829 \\
\hline CTMT3 & 15.5556 & 7.576 & .506 & .861 \\
\hline CTMT4 & 16.0000 & 6.579 & .653 & .827 \\
\hline CTMT5 & 15.8796 & 6.387 & .825 & .783 \\
\hline
\end{tabular}

Nguồn: Kết quả nghiên cứu của tác giả

\subsection{Phân tích nhân tố khám phá EFA}

Phân tích nhân tố khám phá $\mathrm{EFA}$ được sử dụng nhằm phân tích tương quan giữa các biến với nhau và rút thành một tập hợp các yếu tố có ý nghĩa. Sau khi kiểm định Cronbach's Alpha và loại đi biến Nhóm yếu tố Pháp lý là biến có tương quan biến - tổng không phù hợp, nghiên cứu tiến hành phân tích nhân tố khám phá EFA, kết quả như sau:

\section{Bảng 2}

Kết quả KMO và Bartlett's test

\begin{tabular}{|l|l|}
\hline \multicolumn{2}{|c|}{ KMO và Bartlett's test } \\
\hline Kaiser-Meyer-Olkin Measure of Sampling Adequacy & .796 \\
\hline Approx. Chi-Square & 809.158 \\
\hline Df & 153 \\
\hline Sig. & .000 \\
\hline
\end{tabular}

Nguồn: Kết quả nghiên cứu của tác giả 
Hệ số $\mathrm{KMO}=0,796>0,5$ cho thấy dữ liệu nghiên cứu có thể sử dụng để phân tích nhân tố được. Kết quả kiểm định Bartlett's với mức ý nghĩa Sig. $=0.000<0,05$. Bác bỏ giả thuyết H0 (các biến không có tương quan với nhau), và như vậy thì các biến có tương quan với nhau trong tổng thể.

\section{Bảng 3}

Kết quả giải thích phương sai

\begin{tabular}{|c|c|c|c|c|c|c|}
\hline \multirow[b]{2}{*}{$\underset{t}{\text { Componen }}$} & \multicolumn{3}{|c|}{ Initial Eigenvalues } & \multicolumn{3}{|c|}{$\begin{array}{c}\text { Extraction Sums of Squared } \\
\text { Loadings }\end{array}$} \\
\hline & Total & $\begin{array}{c}\% \text { of } \\
\text { Variance }\end{array}$ & $\underset{\%}{\text { Cumulative }}$ & Total & $\begin{array}{c}\% \text { of } \\
\text { Variance }\end{array}$ & $\underset{\%}{\text { Cumulative }}$ \\
\hline 1 & 5.745 & 31.917 & 31.917 & 5.745 & 31.917 & 31.917 \\
\hline 2 & 2.342 & 13.009 & 44.925 & 2.342 & 13.009 & 44.925 \\
\hline 3 & 1.377 & 7.648 & 52.574 & 1.377 & 7.648 & 52.574 \\
\hline 4 & 1.276 & 7.089 & 59.663 & 1.276 & 7.089 & 59.663 \\
\hline 5 & .983 & 5.462 & 65.124 & & & \\
\hline 6 & .892 & 4.954 & 70.078 & & & \\
\hline 7 & .756 & 4.198 & 74.275 & & & \\
\hline 8 & .693 & 3.851 & 78.127 & & & \\
\hline 9 & .641 & 3.561 & 81.688 & & & \\
\hline 10 & .579 & 3.218 & 84.905 & & & \\
\hline 11 & .541 & 3.005 & 87.911 & & & \\
\hline 12 & .520 & 2.889 & 90.800 & & & \\
\hline 13 & .425 & 2.364 & 93.164 & & & \\
\hline 14 & .350 & 1.947 & 95.111 & & & \\
\hline 15 & .321 & 1.786 & 96.897 & & & \\
\hline 16 & .264 & 1.469 & 98.366 & & & \\
\hline 17 & .153 & .851 & 99.216 & & & \\
\hline 18 & .141 & .784 & 100.000 & & & \\
\hline
\end{tabular}

Nguồn: Kết quả nghiên cứu của tác giả 


\section{Bảng 4}

Kết quả phân tích nhân tố khám phá EFA

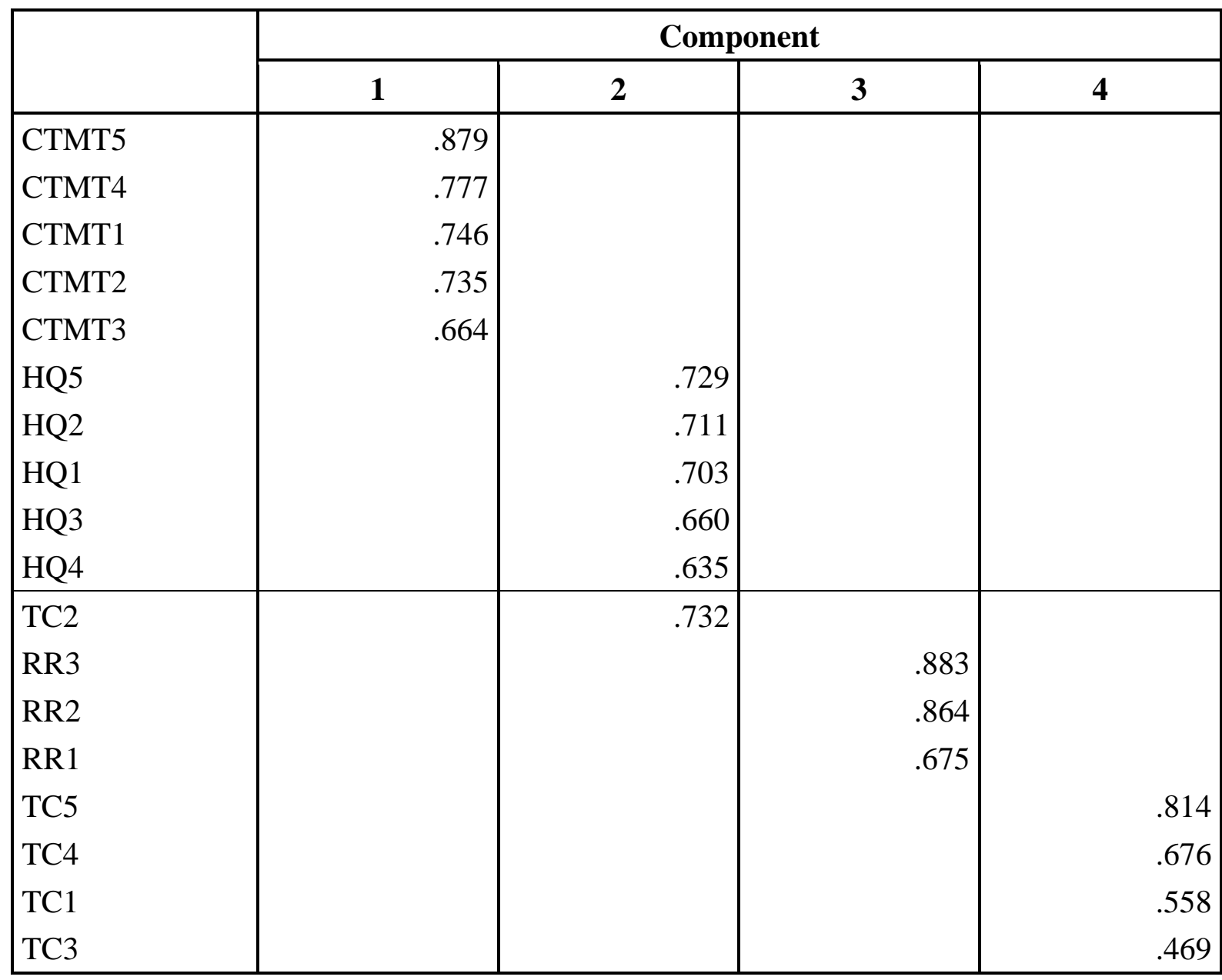

Nguồn: Kết quả nghiên cứu của tác giả

Kết quả phân tích nhân tố khám phá cũng cho thấy tại Eigenvalues $>1$ thì phân tích nhân tố đã trích được 4 yếu tố từ 18 biến quan sát. Phương sai trích đạt $59,663 \%$ thể hiện rằng yếu tố được rút ra giải thích được 59,663\% biến thiên dữ liệu (>50\%). Như vậy có thể kết luận thang đo rút ra là chấp nhận được.

\subsection{Chỉ số quan trọng tưong đối (Relative Importance Index - RII)}

Sau khi phân tích nhân tố khám phá và loại biến Nhóm yếu tố Pháp lý sau kiểm định Cronbach's Alpha, nghiên cứu tính toán chỉ số quan trọng tương đối (Relative Importance Index - RII) đối với từng yếu tố trong nhóm yếu tố, để xác định mức độ tác động của từng yếu tố trong nhóm yếu tố tới việc hoàn thành dự án PPP. Kết quả đạt được như sau:

Trong Nhóm yếu tố Quản lý rủi ro, yếu tố RR3: Các rủi ro về giảm lợi nhuận đối với khu vực tư nhân, được đánh giá là ảnh hưởng nhiều nhất trong nhóm yếu tố Quản lý rủi ro đối với việc hoàn thành dự án PPP. Thực tế, đối với việc kêu gọi đầu tư các dự án PPP tại TP.HCM, điều mà các nhà đầu tư quan tâm nhất là khả năng đảm bảo lợi nhuận cho khu vực tư, khi mà dự án PPP thường là các dự án dài hạn, có số vốn đầu tư ban đầu rất lớn và ở những lĩnh vực 
có lợi nhuận dự kiến không cao. Yếu tố RR2: Các rủi ro về tài chính (lãi suất, tỷ giá, lạm phát,...) lại ảnh hưởng yếu nhất trong dự án PPP, điều này tương đối hợp lý đối với sự ổn định của kinh tế vĩ mô tại Việt Nam.

Trong nhóm yếu tố Hiệu quả trong quá trình thực hiện dự án, Yếu tố HQ1: Các thiết kế rõ ràng, dễ hiểu và đủ thông tin cho các bên thực hiện được đánh giá là tác động nhiều nhất trong nhóm yếu tố Hiệu quả trong quá trình thực hiện dự án. Tiếp theo là HQ3: Chất lượng của các nhà tư vấn và nhà thầu thực hiện dự án và $\mathrm{HQ}$ : Có sự hỗ trợ của các bên tham gia trong quá trình phát triển dự án. Như vậy có thể thấy rằng, việc đảm bảo thông tin và phối hợp công việc giữa Chủ đầu tư, các nhà tư vấn, nhà thầu là điểm quan trọng nhất ảnh hưởng tới việc hoàn thành dự án PPP. Yếu tố HQ2: Áp dụng các công nghệ xây dựng mới được đánh giá là ít ảnh hưởng nhất tới việc hoàn thành dự án PPP, điều này có thể do các dự án PPP tại TP.HCM với sự tham gia của tư nhân chưa có khai thác nhiều thế mạnh về công nghệ của khối tư nhân, mà thường nhằm mục đích giải quyết thiếu hụt vốn trong đầu tư cơ sở hạ tầng hoặc xử lý rác thải, môi trường.

Trong nhóm yếu tố Tài chính dự án, Yếu tố TC3: Khả năng tiết kiệm trong tổng chi phí dự án và yếu tố TC4: Giải quyết các vấn đề hạn chế về ngân sách nhà nước, được đánh giá là 2 yếu tố ảnh hưởng nhiều nhất tới việc hoàn thành dự án PPP ở góc độ Tài chính dự án. Như vậy các dự án PPP với mục đích huy động nguồn lực tư nhân thông qua cơ chế hợp tác với khu vực tư, vẫn cần phải đảm bảo kỷ luật ngân sách như chi tiêu công nhưng phải đảm bảo giải quyết được những hạn chế về ngân sách mà khu vực công gặp phải.

Trong nhóm yếu tố Chính trị và môi trường kinh doanh, yếu tố CTMT3: Sự trung thực trong thực hiện dự án được đánh giá là ảnh hưởng nhiều nhất. Bên cạnh đó, yếu tố CTMT5: Các vấn đề xã hội và sự ủng hộ của cộng đồng cũng là một trong những yếu tố quan trọng ảnh hưởng tới dự án.

\section{Bảng 5}

RII của các yếu tố trong nghiên cứu

\begin{tabular}{|c|c|c|c|c|c|c|c|c|}
\hline \multirow{2}{*}{ Biến quan sát } & \multicolumn{5}{|c|}{ Tần suất được lựa chọn bởi mẫu } & \multirow[t]{2}{*}{ Mức ý nghĩa } & \multirow[t]{2}{*}{ RII } & \multirow[t]{2}{*}{ Xếp hạng } \\
\hline & "5" & “4” & “3” & "2" & “1” & & & \\
\hline RR1 & 22 & 58 & 26 & 2 & 0 & 3.926 & 0.785 & 2nd \\
\hline RR2 & 23 & 51 & 31 & 3 & 0 & 3.870 & 0.774 & 3rd \\
\hline RR3 & 42 & 58 & 8 & 0 & 0 & 4.315 & 0.863 & $1 \mathrm{st}$ \\
\hline HQ1 & 20 & 72 & 15 & 1 & 0 & 4.028 & 0.806 & 1 st \\
\hline HQ2 & 19 & 48 & 35 & 6 & 0 & 3.741 & 0.748 & 5th \\
\hline HQ3 & 28 & 54 & 19 & 7 & 0 & 3.954 & 0.791 & 2nd \\
\hline HQ4 & 25 & 47 & 27 & 9 & 0 & 3.815 & 0.763 & 4th \\
\hline $\mathrm{TC} 1$ & 8 & 75 & 22 & 3 & 0 & 3.815 & 0.763 & $3 \mathrm{rd}$ \\
\hline TC2 & 25 & 37 & 39 & 7 & 0 & 3.741 & 0.748 & 5th \\
\hline TC3 & 22 & 56 & 28 & 2 & 0 & 3.907 & 0.781 & $1 \mathrm{st}$ \\
\hline TC4 & 28 & 42 & 29 & 9 & 0 & 3.824 & 0.765 & 2nd \\
\hline
\end{tabular}




\begin{tabular}{|c|c|c|c|c|c|c|c|c|}
\hline \multirow{2}{*}{ Biến quan sát } & \multicolumn{5}{|c|}{ Tần suất được lựa chọn bởi mẫu } & \multirow[t]{2}{*}{ Mức ý nghĩa } & \multirow[t]{2}{*}{ RII } & \multirow[t]{2}{*}{ Xếp hạng } \\
\hline & "5" & "4" & "3" & "2" & "1" & & & \\
\hline TC5 & 17 & 57 & 29 & 4 & 1 & 3.787 & 0.757 & 4th \\
\hline CTMT1 & 19 & 61 & 25 & 3 & 0 & 3.889 & 0.778 & $3 \mathrm{rd}$ \\
\hline СТMT2 & 30 & 38 & 31 & 9 & 0 & 3.824 & 0.765 & 5 th \\
\hline СТMT3 & 50 & 41 & 17 & 0 & 0 & 4.306 & 0.861 & $1 \mathrm{st}$ \\
\hline СTMT4 & 24 & 52 & 26 & 5 & 1 & 3.861 & 0.772 & 4th \\
\hline СТMT5 & 28 & 52 & 26 & 2 & 0 & 3.981 & 0.796 & $2 \mathrm{nd}$ \\
\hline
\end{tabular}

Nguồn: Kết quả nghiên cứu của tác giả

\section{Kết luận và khuyến nghị}

\subsection{Kết luận}

Trên nền tảng nghiên cứu của các tác giả trước đã được lược khảo chi tiết, nghiên cứu này chỉ ra rằng các nhóm yếu tố (1) Quản lý rủi ro; (2) Hiệu quả trong quá trình thực hiện dự án; (3) Tài chính dự án; (4) Chính trị và môi trường kinh doanh là các nhóm yếu tố ảnh hưởng tới việc hoàn thành dự án PPP tại Thành phố Hồ Chí Minh. Trong đó, đối với từng nhóm yếu tố, thì các yếu tố: Các rủi ro về giảm lợi nhuận đối với khu vực tư nhân; Các thiết kế rõ ràng, dễ hiểu và đủ thông tin cho các bên thực hiện; Khả năng tiết kiệm trong tổng chi phí dự án; Sự trung thực trong thực hiện dự án là các yếu tố được đánh giá là ảnh hưởng nhiều nhất. Về mặt lý thuyết, nghiên cứu này bổ sung và tiếp nối các nghiên cứu nền tảng trên thế giới về việc hoàn thành dự án PPP. Về thực tiễn, nghiên cứu chỉ ra những yếu tố ảnh hưởng nhiều tới dự án, để từ đó các bên tham gia dự án (Cơ quan Quản lý nhà nước, Nhà đầu tư,...) có thêm cơ sở để điều chỉnh và đưa ra những khuyến nghị chính sách để các dự án sắp tới được thực hiện tốt hơn.

\subsection{Khuyến nghị chính sách}

\subsection{1. Đối với khía cạh Quản lý rủi ro}

Đối với khía cạnh liên quan đến rủi ro, trong dự án cần phải cụ thể hóa các bên chịu những rủi ro trong quá trình thực hiện, và có chế tài nếu vi phạm nhằm bảo vệ quyền lợi cho cả nhà nước và nhà đầu tư. Các chế tài và cơ chế phân bổ rủi ro này cần được quy định rõ trong hợp đồng PPP theo giao kết ban đầu, hoặc hình thành bộ quy tắc phân bổ rủi ro đối với từng loại dự án theo luật định. Đặc biệt, cần có chính sách để giảm thiểu những rủi ro liên quan đến lợi nhuận dự án. Có thể là các cam kết, các cơ chế bảo lãnh đặc thù cho các rủi ro về lưu lượng, doanh thu, chuyển đổi ngoại tệ, etc. hoặc Nhà nước bỏ ra các nguồn vốn đối ứng từ ngân sách hoặc theo hình thức trái phiếu công trình để đảm bảo việc vận hành và lợi nhuận cho các dự án PPP dài hạn, nhất là trong lĩnh vực đầu tư cơ sở hạ tầng như đường bộ cao tốc, tàu điện ngầm đô thị, đường sắt tốc độ cao,...

\subsection{2. Đối với khía cạnh Hiệu quả trong quá trình thực hiện dụ án}

Các yếu tố: Các thiết kế rõ ràng, dễ hiểu và đủ thông tin cho các bên thực hiện và Chất lượng của các nhà tư vấn và nhà thầu thực hiện dự án được đánh giá cao nhất đối với việc hoàn thành dự án PPP. Như vậy chính sách của Nhà nước cần đẩy mạnh hơn nữa việc đảm bảo thông tin của dự án và chất lượng nhà thầu tham gia. Cần cụ thể hóa việc nâng cao hiệu quả dự án này thông qua việc xây dựng một hành lang pháp lý ổn định, với các Luật, Nghị định, Thông tư và các hướng dẫn thực thi khác rõ ràng để cả Nhà nước và Nhà đầu tư thực hiện. Các quy định về 
dự án PPP cũng cần bổ sung thêm yêu cầu công khai thông tin về dự án PPP trong suốt vòng đời dự án, bao gồm cả thông tin về tiến độ thực hiện để tạo môi trường thông tin thông suốt cho các nhà đầu tư cũng như đảm bảo sự tham gia giám sát của cộng đồng.

\subsection{3. Đối với khía cạnh Tài chính dự án}

Ở khía cạnh Tài chính dự án thì yếu tố: Khả năng tiết kiệm trong tổng chi phí dự án và yếu tố: Giải quyết các vấn đề hạn chế về ngân sách nhà nước, được đánh giá là 2 yếu tố ảnh hưởng nhiều nhất tới việc hoàn thành dự án PPP. Như vậy, phần lớn những người tham gia khảo sát đều hiểu rằng các dự án PPP được thực hiện nhằm mục đích giảm gánh nặng cho ngân sách nhà nước. Tuy nhiên, nhiều nghiên cứu về các suất đầu tư dự án PPP tại Trung Quốc cho thấy rằng không hẳn việc đầu tư PPP là có hiệu quả về mặt tài chính hơn là đầu tư công thông thường. Điều này đặt ra một vấn đề là Nhà nước cần xác định khi có 1 dự án cần đầu tư, ví dụ như xây dựng kết cấu hạ tầng, thì khi muốn chuyển phương án đầu tư công sang đầu tư PPP cần phải có các tính toán hợp lý để không gây thiệt hại đến lợi ích của người dân và người tiêu dùng.

Về giải pháp tài chính, nhằm có sự minh bạch trong quá trình thực hiện dự án và cũng hạn chế rủi ro tài chính, cần đa dạng hóa nguồn tài chính, bao gồm vốn chủ đầu tư ban đầu, vốn vay thương mại, vốn trái phiếu công trình và vốn từ các nguồn khác (quỹ đầu tư, cổ đông vãng lai). Các cơ chế đa bên với sự tham gia của nhiều tổ chức, nhiều góc độ, sẽ tạo ra sự đàm phán, thẩm định và giám sát tập thể, khách quan và minh bạch đối với dự án, thay cho cơ chế thỏa thuận hai bên là chủ đầu tư và cơ quan quản lý nhà nước như hiện nay.

\subsection{4. Đối với khía cạnh Chính trị và môi trường kinh doanh}

Yếu tố: Sự trung thực trong thực hiện dự án được đánh giá là ảnh hưởng nhiều nhất trong nhóm yếu tố Chính trị và môi trường kinh doanh. Điều này gợi ý cho những nhà làm chính sách cần minh bạch hóa và chuyên nghiệp hóa việc thực hiện dự án PPP. Đối với việc minh bạch hóa, các dự án PPP nên thực thi theo các thông lệ quốc tế nhằm tận dụng được các cơ chế hợp tác và minh bạch mà các nước phát triển đã sử dụng, đồng thời cũng là cơ hội thu hút các nhà đầu tư nước ngoài. Các dự án PPP cũng nên có quá trình tham vấn cộng đồng, nhằm có thêm các bên giám sát, đảm bảo sự minh bạch và trung thực trong quá trình hợp tác, hài hòa lợi ích của các bên liên quan.

\section{Hạn chế và hướng nghiên cứu tiếp theo}

Ngoài những kết quả nghiên cứu đã được trình bày, nghiên cứu này vẫn còn tồn tại những hạn chế cần bổ sung và hoàn thiện đối với việc nghiên cứu các yếu tố tác động tới việc hoàn thành dự án PPP tại Thành phố Hồ Chí Minh, cụ thể:

Thứ nhất, nghiên cứu này được tiến hành thông qua việc thu thập dữ liệu trong thời gian rất ngắn là 06 tháng và phân tích trên 108 phản hồi, là một số lượng mẫu tương đối nhỏ so với quy mô của TP.HCM và các dự án $\mathrm{PPP}$, điều này có thể dẫn tới việc không phản ảnh được hết kết quả nghiên cứu. Các nghiên cứu tiếp theo cần có thời gian thu thập dữ liệu dài hơn với quy mô mẫu lớn hơn, nhằm phản ánh tốt hơn về kết quả nghiên cứu.

Thứ hai, nghiên cứu này chỉ sử dụng các phương pháp thống kê mô tả, phân tích nhân tố và tính toán chỉ số quan trọng tương đối - RII, chưa phân tích được liệu các biến độc lập có tác động tới nhau hay không. Ngoài ra các phương pháp này cũng chưa xác định được tính chất và mức độ quan trọng của các biến nghiên cứu. Các nghiên cứu tiếp theo có thể xây dựng thêm bảng hỏi cho biến phụ thuộc và sử dụng phân tích hồi quy khi nghiên cứu. 


\section{Tài liệu tham khảo}

Akintoye, A., Craig, T., \& Eamon, F. (1998). Risk analysis and management of private finance initiative projects. Engineering, Construction and Architectural Management, 5(1), 9-21.

Beyene, T. (2014). Factors for Implementing Public-Private Partnership (PPP) in the development process: Stakeholders' perspective from Ethiopia. International Journal of Science and Research, 3(3), 792-797.

Chan, A. P., Lam, P. T. I., Chan, D. W. M., Cheung, E., \& Ke, Y. (2010). Critical success factors for PPPs in infrastructure developments: Chinese perspective. Journal of Construction Engineering and Management, 136(5), 484-494.

Cheung, E., Chan, A. P. C., \& Kajewski, S. (2009). Reasons for implementing public private partnership projects: Perspectives from Hong Kong, Australian and British practitioners. Journal of Property Investment \& Finance, 27(1), 81-95. doi:10.1108/14635780910926685

European Commission. (2003). Guidelines for successful public - Private partnerships. $\begin{array}{llll}\text { Retrieved } \quad \text { September } & \text { 20, from }\end{array}$ https://ec.europa.eu/regional_policy/sources/docgener/guides/ppp_en.pdf

European PPP Expertise Centre. (2010). The guide to guidance: How to prepare, procure and deliver PPP projects. Retrieved September 21, 2018, from https://www.utap.gov.pt/Publicacoes_interna/guide-to-guidance-en.pdf

Ghobadian, A., O’Regan, N., Gallear, D., \& Viney, H. (2004). Public-private partnerships: Policy and experience. Basingstoke, UK: Palgrave Macmillan.

Grimsey, D., \& Lewis, M. K. (2002). Evaluating the risks for public private partnerships for infrastructure projects. International Journal of Project management, 20(2), 107-118.

Huynh, G. T. T. (2012). Hình thức hợp tác công tu (Public Private Partnership) để phát triển cơ sở hạ tầng giao thông đường bộ tại Việt Nam [Public Private Partnership to develop road transport infrastructure in Vietnam] (Doctoral dissertation). Trường Đại học Kinh tế Thành phố Hồ Chí Minh, Việt Nam.

Hair, J. F., Black, W. C., Babin, B. J., Anderson, R. E., \& Tatham, R. L. (2006). Multivariate data analysis (Vol. 6). Upper Saddle River, NJ: PearsonPrentice Hall.

Hardcastle, C., Edwards, P. J., Akintoye, A., \& Li, B. (2005). Critical success factors for PPP/PFI projects in the UK construction industry. Construction Management and Economics, 23(5), 459-471.

Ismail, S. (2013). Critical success factors of public private partnership (PPP) implementation in Malaysia. Asia-Pacific Journal of Business Administration, 5(1), 6-19.

Ismail, S., \& Ajija, S. R. (2012). Critical success factors of public private partnership (PPP) implementation in Malaysia. Jurnal Akuntansi dan Keuangan Indonesia, 4(2), 137-154.

Kwak, Y. H., Chih, Y., \& Ibbs, C. W. (2009). Towards a comprehensive understanding of public private partnerships for infrastructure development. California Management Review, 51(2), 51-78. 
Li, B., Akintoye, A., Edwards, P. J., \& Hardcastle, C. (2005). Critical success factors for PPP/PFI projects in the UK construction industry. Construction Management and Economics, 23(5), 459-471.

Li, J., \& Zou, P. (2012). Risk identification and assessment in PPP infrastructure projects using fuzzy analytical hierarchy process and life-cycle methodology. Australasian Journal of Construction Economics and Building, 8(1), 34-48.

Mouraviev, N. (2012). Risk management in public-private partnerships and research agenda for transitional countries. International Journal of Management \& Business Studies, 2(2), $27-41$

Tang, L. Y., Shen, Q., Skitmore, M., \& Cheng, E. W. L. (2013). Ranked critical factors in PPP briefings. Journal of Management in Engineering, 29(2), 164-171.

Vassallo Magro, J. M., \& Bartolomé, R. I. (2010). Infraestructura pública y participación privada: Conceptosy experiencias en América y España. Retrieved September 22, 2018, from http://scioteca.caf.com/handle/123456789/421

Walker, C. T., \& Smith, A. J. (1995). Privatized infrastructure: The build operate transfer approach. London, UK: Thomas Telford.

Wang, X. (2017). Risk management of PPP projects in China (Dissertation No. 482). Retrieved September 23, 2018, from http://urn.kb.se/resolve?urn=urn:nbn:se:kth:diva-211147

Yescombe, E. (2007). Public-private partnerships: Principles of policy and finance. Amsterdam: Elsevier Butterworth-Heinemann.

Yitmen, I., Akiner, I., \& Tekce, I. (2012). Applicability of second generation public private partnership projects in turkey: A conceptual framework. Paper presented at the International Conference on Innovation in Architecture, Engineering \& Construction, Sao Paulo, Brasil.

Yuan, J., Wang, C., Skibniewski, M. J., \& Li, Q. (2011). Developing key performance indicators for public-private partnership projects: Questionnaire survey and analysis. Journal of Management in Engineering, 28(3), 252-264.

Zhang, X. (2005). Critical success factors for public-private partnerships in infrastructure development. Journal of construction engineering and management, 131(1), 3-14.

Zhang, X. (2005). Criteria for selecting the private-sector partner in public-private partnerships. Journal of construction engineering and management, 131(6), 631-644. 